\title{
Arecibo Discovery of the Pre-biotic Molecule Methanimine in Arp 220 and New cm-Wavelength Transitions of Other Molecules
}

\author{
E. Momjian ${ }^{* a b}$, C. J. Salter ${ }^{a}$, T. Ghosh ${ }^{a}$, M. S. Lerner ${ }^{a}$, R. Minchin ${ }^{a}$, B. Catinella ${ }^{c}$, M. \\ Lebron $^{d}$ \\ ${ }^{a}$ NAIC, Arecibo Observatory \\ ${ }^{b} N R A O$ \\ ${ }^{c}$ MPIfA \\ ${ }^{d}$ University of Puerto Rico \\ E-mail: emomjian@nrao.edu, csalter@naic.edu, tghosh@naic.edu \\ lerner@naic.edu, rminchin@naic.edu bcatinel@mpa-garching.mpg.de \\ mlebronenaic.edu
}

\begin{abstract}
An on-going Arecibo line search between 1.1 and $10 \mathrm{GHz}$ of the prototypical starburst/megamaser galaxy, Arp 220, has revealed a spectrum rich in molecular transitions. These include the prebiotic molecules: methanimine $\left(\mathrm{CH}_{2} \mathrm{NH}\right)$ in emission, three $v_{2}=1$ direct l-type absorption lines of $\mathrm{HCN}$, and an absorption feature from either ${ }^{18} \mathrm{OH}$ or formic acid $(\mathrm{HCOOH})$. In addition, we report three transitions of $\lambda 4-\mathrm{cm}$ excited $\mathrm{OH}$ not previously detected in Arp 220 which are seen in absorption, and a possible absorption feature from the $6.668 \mathrm{GHz}$ line of methanol. Our results mark the first distant extragalactic detection of methanimine, a molecule with high relevance to the origins of life. Further, the strong, previously undetected, cm-wave $\mathrm{HCN} v_{2}=1$ direct l-type lines can aid the study of dense molecular gas and active star-forming regions in this starburst galaxy.
\end{abstract}

From planets to dark energy: the modern radio universe

October 1-5 2007

University of Manchester, Manchester, UK

* Speaker. 
According to current views, interstellar organic species mostly form on the surface of dust grains, and heating events, such as the formation of a protostar, release the icy grain mantles into the gas phase. These molecules may then form amino acids by the combination of organic species known as pre-biotic molecules. Methanimine $\left(\mathrm{CH}_{2} \mathrm{NH}\right)$ is one such molecule [1] and has been detected in the interstellar medium of our own Galaxy [2] 3] and tentatively in the nearby galaxy, NGC 253 [ \], but never beyond the neighborhood of our Galaxy (i.e. beyond 5 Mpc).

At a distance of $\sim 77 \mathrm{Mpc}$ (redshift, $z=0.018126$ ), Arp 220 is the nearest Ultra-Luminous Infra-Red Galaxy (ULIRG) and the prototypical OH megamaser galaxy. At Arecibo, we are conducting a spectral survey of Arp 220 between 1.1 and $10 \mathrm{GHz}$, for which the initial observations took place between 31 March and 22 April, 2007. Here we report the discovery of various molecular transitions as listed below.

\section{Methanimine $\left(\mathrm{CH}_{2} \mathrm{NH}\right)$}

A broad emission feature covering all six $1_{10}-1_{11}$ multiplet transitions of methanimine is presented in Fig. 1(a). The total velocity width (FWHM) of this line is $270 \mathrm{~km} \mathrm{~s}^{-1}$, and the derived lower limit to the brightness temperature is $\sim 2800 \mathrm{~K}$. Taking into account the multiple components, this drops to $\sim 1000 \mathrm{~K}$, which is similar to the methanimine decomposition temperature of $1300 \mathrm{~K}$ [5]. If the emission solid angle is smaller, then the brightness temperature could be much higher. We conclude that, as for formaldehyde [6], methanimine in Arp 220 is likely to be showing weak maser emission for this transition. High angular resolution observations are underway to address this issue.

\section{Hydrogen Cyanide (HCN)}

In Fig. 1(b,c,d), we present the spectra of the $v_{2}=1$ direct l-type transitions of HCN in the $\mathrm{J}=4,5$ and 6 vibrational levels, which are seen in absorption toward Arp 220. We note that these transitions seem not to have been previously detected in any celestial source.

\section{Excited OH}

Our observations have also detected $\lambda 6$-, 5- and 4-cm $\Lambda$-doublet transitions of the $\mathrm{OH}$ radical seen in absorption against the continuum emission of Arp 220. The $\lambda 6$ - and 5-cm lines have been previously detected by [7] and [ह], respectively. The three $\lambda 4-\mathrm{cm} \mathrm{OH}$ lines are detected for the first time and show similar velocity widths to most other molecular species in Arp 220. Fig. 1(e,f) shows the spectra of the two $\lambda 4-\mathrm{cm}$ excited $\mathrm{OH}$ transitions $2 \Pi_{1 / 2}, \mathrm{~J}=3 / 2, \mathrm{~F}=1-1$ and $2-2$.

\section{Formic Acid (HCOOH) or ${ }^{18} \mathrm{OH}$}

In Fig. 1(g), we present a high signal-to-noise detection of an absorption line at L-band. Despite the presence of nearby Radio Frequency Interference (RFI) caused by Glonass emissions, the reality of this absorption line has been verified by its presence in each of 13 individual scans of Arp 220, and its absence in the scans of the bandpass calibrator. As shown in the figure, there is an 

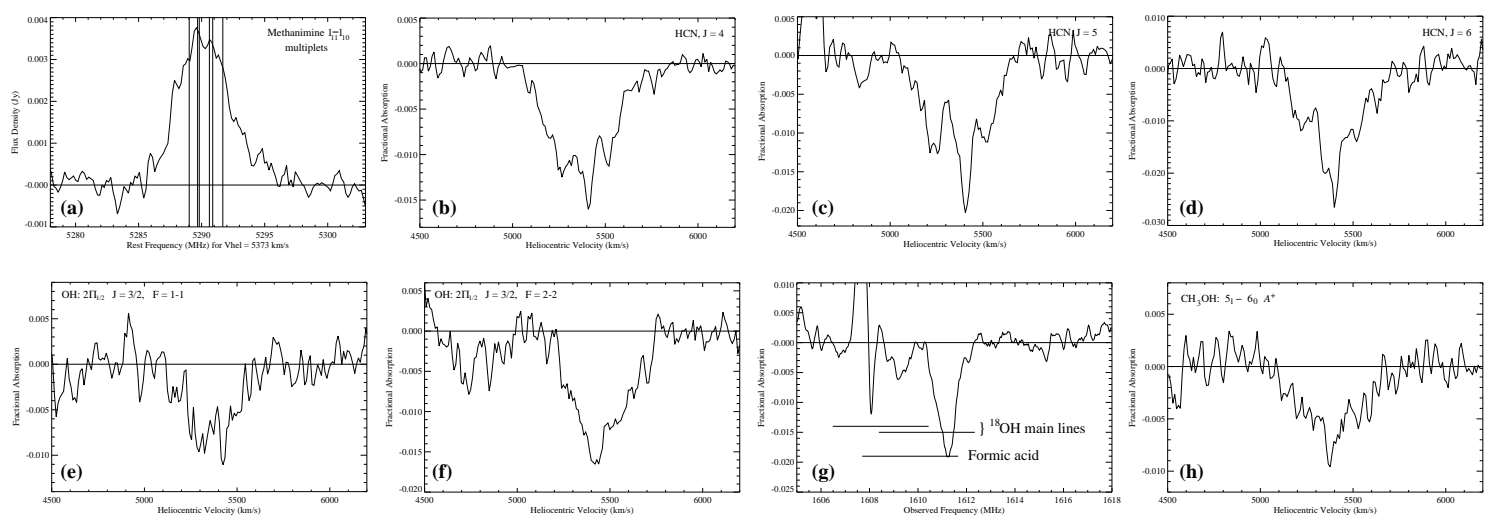

Figure 1: (a): The blended emission line from the six $1_{10}-1_{11}$ multiplet transitions of methanimine in Arp 220. (b, c, d): Spectra of the first astronomical detection of the the $v_{2}=1$ direct l-type absorption lines of $\mathrm{HCN}$ with vibrational levels $\mathrm{J}=4,5$ and 6 . (e, f): First detection of the two $\lambda 4$-cm excited $\mathrm{OH}$ transitions $2 \Pi_{1 / 2}, \mathrm{~J}=3 / 2, \mathrm{~F}=1-1$ and 2-2. (g): One (or possibly two) absorption line(s) that could be either formic acid or ${ }^{18} \mathrm{OH}$. The band is affected by interference from the Glonass system at about $1605 \mathrm{MHz}$. (h): A possible detection of the $55_{1}-6_{0} A^{+}$methanol line in absorption.

ambiguity as to the molecule responsible for this absorption, which could be either ${ }^{18} \mathrm{OH}$ or formic acid $(\mathrm{HCOOH})$. Given the strong prevalence of $\mathrm{OH}$ in Arp 220, it is perhaps not unreasonable to detect the presence of ${ }^{18} \mathrm{OH}$ as well. However, since formic acid is involved in the chemical orgin of life, it would be most interesting to resolve this ambiguity.

\section{Methanol $\left(\mathrm{CH}_{3} \mathrm{OH}\right)$}

In Fig 1(h), we present a possible detection of the $5_{1}-6_{0} A^{+}$methanol line $(6.668 \mathrm{GHz})$ in absorption. Although this line is apparently detected with a low signal-to-noise ratio, and the entire $100-\mathrm{MHz}$ band in which it is observed is basically RFI-free, the quality of the spectral baseline in this case is rather poor. We await our remaining observations to confirm the reality of this detection.

\section{References}

[1] Kirchoff, W.H., Johnson, D. R., \& Lovas, F.J. 1973, J. Phys. Chem. Ref. Data, 2, 1

[2] Godfrey, P.D., Brown, R.D., Robinson, B.J., \& Sinclair, M.W. 1973, Astrophys. Lett., 13, 119

[3] Dickens, J.E., Irvine, W.M., DeVries, C.H., \& Ohishi, M. 1997, ApJ, 479, 307

[4] Martín, S., et al. 2006, ApJS, 164, 450

[5] Nguyen, M.T., Sengupta, D., \& Ha, T-K. 1996, J. Phys. Chem., 100, 6499

[6] Araya, E., Baan, W.A., \& Hofner, P. 2004, ApJS, 154, 541

[7] Henkel, C., Güsten, R., \& Batrla, W., 1986, A\&A, 168, L13

[8] Henkel, Güsten \& Baan 1987, A\&A, 185, 14 